International Journal of Pure and Applied Mathematics

Volume $97 \quad$ No. 2 2014, 241-251

ISSN: $1311-8080$ (printed version); ISSN: 1314-3395 (on-line version)

url: http://www.ijpam.eu

doi: http://dx.doi.org/10.12732/ijpam.v97i2.13

ijpam.eu

\title{
GENERALIZED HAMMING WEIGHTS OF DUALS OF ALGEBRAIC-GEOMETRIC CODES
}

\author{
E. Ballico \\ Department of Mathematics \\ University of Trento \\ 38123 Povo (Trento) - Via Sommarive, 14, ITALY
}

\begin{abstract}
Here we extends a work of A. Couvreur on the Hamming distance of the dual of an evaluation code to its generalized Hamming weights. We prove the following result.

Fix integers $r \geq 2, m>0$ and $e \geq 1$. Let $Z \subset \mathbb{P}^{r}$ be a zero-dimensional scheme such that $\operatorname{deg}(Z) \leq 3 m+r-3$. If $r>2$ assume that $Z$ spans $\mathbb{P}^{r}$ and that the sum of the degrees of the non-reduced connected components of $Z$ is at most $2 m+1$. We have $h^{1}\left(\mathcal{I}_{Z}(m)\right) \geq e$ if and only if there is $W \subseteq Z$ as one of the schemes in the following list:

(a) $\operatorname{deg}(W)=m+1+e$ and $W$ is contained in a line;

(b) $\operatorname{deg}(W)=2 m+1+e$ and $W$ is contained in a reduced plane conic;

(c) $r \geq 3, e \geq 2$, and there are an integer $f \in\{1, \ldots, e-1\}$ and lines $L_{1}, L_{2}$, such that $L_{1} \cap L_{2}=\emptyset, \operatorname{deg}\left(L_{1} \cap Z\right)=m+1+f$ and $\operatorname{deg}\left(L_{2} \cap Z\right)=$ $m+1+e-f$.
\end{abstract}

AMS Subject Classification: 4N05, 14Q05, 94B27

Key Words: dual code, generalized Hamming weights, higher support weights, evaluation code, plane curve, algebraic-geometric code 


\section{Introduction}

Fix a prime $p$ and a $p$-power $q$. We recall that an affine $[n, k]$-code $\mathcal{C}$ over $\mathbb{F}_{q}$ is often given in the following way. Fix positive integers $n, r$, distinct points $P_{1}, \ldots, P_{n}$ of the affine space $\mathbb{F}_{q}^{m}$ and a $k$-dimensional linear subspace $W$ of $\mathbb{F}_{q}\left[t_{1}, \ldots, t_{m}\right]$ such that no $f \in W \backslash\{0\}$ vanishes at all points $P_{1}, \ldots, P_{n}$. Fix a basis $f_{1}, \ldots, f_{k}$ of $W$. The $k \times n$ matrix $\left(f_{i}\left(P_{j}\right)\right)$ gives an injective linear map $\mathbb{F}^{k} \rightarrow \mathbb{F}_{q}^{n}$, i.e., this matrix is the generator matrix of an $[n, k]$-code $\mathcal{C}([11])$. he dual code $\mathcal{C}^{\vee}$ is the $[n,(n-k)]$-code whose words are the elements of $\mathbb{F}_{q}^{n}$ orthogonal to the words of $\mathcal{C}$ with respect to the canonical inner product, i.e., $\left(a_{1}, \ldots, a_{n}\right) \in \mathbb{F}_{q}^{n}$ is a word of $\mathcal{C}^{\vee}$ if and only if $a_{1} b_{1}+\cdots+a_{n} b_{n}=0$ for all words $\left(b_{1}, \ldots, b_{n}\right)$ of $\mathcal{C}$ (i.e. the generator matrix of $\mathcal{C}^{\vee}$ is the parity check matrix of $\mathcal{C}$, and conversely). We usually consider the following projective set-up, leaving to the interested reader the task to translate the projective language into the affine language. For any field $K$ let $K\left[x_{0}, \ldots, x_{r}\right]_{m}$ denote the set of homogeneous polynomials over $K$ with degree $m$. The $K$-vector space $K\left[x_{0}, \ldots, x_{r}\right]$ has dimension $\left(\begin{array}{c}m+r \\ r\end{array}\right)$. Fix a finite set $S \subseteq \mathbb{P}^{r}\left(\mathbb{F}_{q}\right)$ and a $k$-dimensional linear subspace of $H^{0}\left(\mathbb{P}^{r}, \mathcal{O}_{\mathbb{P}^{r}}(m)\right)$ defined over $\mathbb{F}_{q}$. We fix homogeneous coordinates $x_{0}, \ldots, x_{r}$ of $\mathbb{P}^{r}$, so that we may identify $V$ with a $k$-dimensional linear subspace of $\mathbb{F}_{q}\left[x_{0}, \ldots, x_{r}\right]$. For any $P \in S$ we fix $P^{\prime} \in$ $\mathbb{F}_{q}^{r+1} \backslash\{0\}$ whose equivalence class modulo scalars induces $P$. We use these choices to identify $S$ with a subset of $\mathbb{F}_{q}^{r+1} \backslash\{0\}$ (also called $S$ ). With these choices every $f \in V$ gives a map $S \rightarrow \mathbb{F}_{q}$. Therefore $V$ induces a linear map $V \rightarrow \mathbb{F}_{q}^{S}$. If this map is injective, then we get an $[n, k]$-code $\mathcal{C}$ over $\mathbb{F}_{q}$. If we identify $V$ with a linear subspace of $\mathbb{F}_{q}^{S}$, then we denote with $V^{\perp}$ its orthogonal linear subspace, i.e., the linear subspace of $\mathbb{F}_{q}^{S}$ inducing $\mathcal{C}^{\vee}$. A. Couvreur proved that quite often it is easier to compute the minimum distance of $\mathcal{C}^{\vee}$ and classify all the codewords of $\mathcal{C}^{\vee}$ with small weights (not only the ones with minimum distance) than to solve the corresponding problems for $\mathcal{C}([7],[5])$. As clear from [7] in evaluation codes arising from $H^{0}\left(\mathbb{P}^{n}, \mathcal{O}_{\mathbb{P}}(m)\right)$ very simple objects (lines, small degree plane curves, finite sets which are complete intersections) are often useful. We extend [7] to the higher distances of a code (a.k.a. higher support weights ([10], [12]) (see Lemma 1). In [2] we translated the issue directly on plane curve giving the associated Goppa code. Lemma 1 is more flexible. By Lemma 1 to get the extension it is sufficient to prove the case $e \geq 2$ of the following result.

Theorem 1. Fix integers $r \geq 2, m>0$ and $e \geq 1$. Let $Z \subset \mathbb{P}^{r}$ be a zerodimensional scheme such that $\operatorname{deg}(Z) \leq \max \{3 m+\rho-3,3 m+e-2\}$ where $\rho$ 
is the dimension of the linear span of $Z$. If $\rho>2$ assume that the sum of the degrees of the non-reduced connected components of $Z$ is at most $2 m+1$. We have $h^{1}\left(\mathcal{I}_{Z}(m)\right) \geq e$ if and only if there is $W \subseteq Z$ as one of the schemes in the following list:

(a) $\operatorname{deg}(W)=m+1+e$ and $W$ is contained in a line;

(b) $\operatorname{deg}(W)=2 m+1+e$ and $W$ is contained in a reduced plane conic;

(c) $r \geq 3, e \geq 2$, and there are an integer $f \in\{1, \ldots, e-1\}$ and lines $L_{1}, L_{2}$, such that $L_{1} \cap L_{2}=\emptyset, \operatorname{deg}\left(L_{1} \cap Z\right)=m+1+f$ and $\operatorname{deg}\left(L_{2} \cap Z\right)=$ $m+1+e-f$.

Case (c) is the one arising only in the set-up of generalized Hamming weight, because if $W$ is as in case (c), then there is $W^{\prime} \subsetneq W$ with $h^{1}\left(\mathcal{I}_{W^{\prime}}(m)\right)>0$, and it is $W^{\prime}$, not $W$, that count for the minimum distance.

Remark 1. Assume that $Z$ is defined over a perfect field $K$ and call $\bar{K}$ the algebraic closure of $K$. It is easy to check that we may find $W$ with the additional condition that $W$ and the curve (a line, or a conic or a disjoint union of two lines in cases (a), (b) or (c)) are defined over a finite extension $K^{\prime}$ of $K$ with $\operatorname{deg}\left(\left[K^{\prime}: K\right]\right) \leq 2$. To get $K^{\prime}=K$ in cases (a) and (b) it is sufficient that one of the points of $W_{\text {red }}$ is defined over $K$. In case 2 if the conic $E$ is either smooth or a double line, then it is defined over $K$. If $E$ is a reduced, but a reducible conic to get that $W$ and $E$ are defined over $K$ it is sufficient that two points of $W_{\text {red }}$ are defined over $K$. For the application to coding theory we have $K=\mathbb{F}_{q}$ and, as in [7] and [5], many of the connected components of $Z$ are reduced and defined over $K$.

If $Z$ is just a finite set, then the proofs are easier and (at least if $e=1$ ) (see [1]). Even weaker assumption on $Z$ may be used (as in [3]). Basically in the proof of Theorem 1 we need in some way to handle the case $D=L$ (or in the case of Lemma 3 the case $D^{\prime}=D$ ).

\section{The Proof and a Result in $\mathbb{P}^{2}$}

Lemma 1. Fix $S \subseteq \mathbb{P}^{r}\left(\mathbb{F}_{q}\right)$ and a linear subspace $V \subseteq \mathbb{F}_{q}\left[x_{0}, \ldots, x_{r}\right]_{m}$ and call $\mathcal{C}$ the evaluation code induced by $(V, S)$. Set $n:=\sharp(S)$ and $k:=\operatorname{dim}(V)$. Assume $V(-S)=\{0\}$, i.e., assume that $\mathcal{C}$ is an $[n, k]$-code.

(a) Fix $B \subseteq S$ and an integer $e>0$. There is an e-dimensional linear subspace of $\mathcal{C}^{\vee}=\left(V^{\perp}, S\right)$ with support contained in $B$ if and only if $i(V, B) \geq e$. 
(b) Fix an integer $h \in\{1, \ldots, k-1\} ; d_{h}\left(\mathcal{C}^{\vee}\right)$ is the minimal cardinality of a set $A \subseteq S$ such that $i(V, A)=h$; all these sets $A$ determine the $h$-dimensional linear subspaces of $V^{\perp}$ with minimal support.

Proof. Fix $B \subseteq S$. We write $S=B \sqcup(S \backslash B)$ and identify $\mathbb{F}_{q}^{S}=\left\{S \rightarrow \mathbb{F}_{q}\right\}$ with $\mathbb{F}_{q}^{B} \times \mathbb{F}_{q}^{S \backslash B}$. The linear projection of $\mathbb{F}_{q}^{S}$ onto its factor $\mathbb{F}_{q}^{B}$ and the inclusion $V \hookrightarrow \mathbb{F}_{q}^{S}$ induces an inclusion $V / V(-B) \hookrightarrow \mathbb{F}_{q}^{B}$. Fix $f \in \mathbb{F}_{q}^{S}$ with support on $B$. Since $f$ has support on $B$, we have $\sum_{P \in S} f(P) g(P)=\sum_{P \in B} f(P) g(P)$ for all $g \in \mathbb{F}_{q}^{S}$. We have $i(V, B)=0$ if and only if the evaluations of $V$ at the points of $B$ are linearly independent. In general $i(V, B)$ is the number of independent linear relations among the evaluations of $V$ at the points of $B$. Hence $i(V, B)$ is the dimension of the linear subspace of $\mathcal{C}^{\vee}$ formed by the words with support on $B$. Hence we get part (a). Part (b) follows from part (a).

Remark 2. Let $X$ be any projective scheme and $D$ any effective Cartier divisor of $X$. For any closed subscheme $Z$ of $X$ let $\operatorname{Res}_{D}(Z)$ denote the residual scheme of $Z$ with respect to $D$, i.e. the closed subscheme of $X$ with $\mathcal{I}_{Z}: \mathcal{I}_{D}$ as its ideal sheaf. We have $\operatorname{deg}(Z)=\operatorname{deg}(Z \cap D)+\operatorname{deg}\left(\operatorname{Res}_{D}(Z)\right)$. If $Z$ is a finite set, then $\operatorname{Res}_{D}(Z)=Z \backslash Z \cap D$. For every $L \in \operatorname{Pic}(X)$ we have the exact sequence

$$
0 \rightarrow \mathcal{I}_{\operatorname{Res}_{D}(Z)} \otimes L(-D) \rightarrow \mathcal{I}_{Z} \otimes L \rightarrow \mathcal{I}_{Z \cap D, D} \otimes(L \mid D) \rightarrow 0
$$

From (1) we get

$$
h^{i}\left(X, \mathcal{I}_{Z} \otimes L\right) \leq h^{i}\left(X, \mathcal{I}_{\operatorname{Res}_{D}(Z)} \otimes L(-D)\right)+h^{i}\left(D, \mathcal{I}_{Z \cap D, D} \otimes(L \mid D)\right)
$$

for every integer $i \geq 0$.

Lemma 2. Let $A, B$ be zero-dimensional subschemes of $\mathbb{P}^{r}$ such that $A \subseteq B$. If $h^{1}\left(\mathbb{P}^{r}, \mathcal{I}_{B}(m)\right)=0$, then $h^{1}\left(\mathbb{P}^{r}, \mathcal{I}_{A}(m)\right)=0$.

Proof. Since $B$ is a zero-dimensional scheme, then $h^{1}(B, \mathcal{F})=0$ for every coherent sheaf $\mathcal{F}$ on $B$. Therefore $h^{1}\left(B, \mathcal{I}_{A, B}(m)\right)=0$. Hence the restriction map $H^{0}\left(B, \mathcal{O}_{B}(m)\right) \rightarrow H^{0}\left(A, \mathcal{O}_{A}(m)\right)$ is surjective.

In $\mathbb{P}^{2}$ by far the strongest tool is the following result due to $\mathrm{Ph}$. Ellia and Ch. Peskine ([8], Corollaire 2) (see [8], Remarques (i) at page 116 for a use of it).

Proposition 1. Fix an integer $m \geq 3$ and a zero-dimensional scheme $Z \subset \mathbb{P}^{2}$ such that either $\operatorname{deg}(Z) \leq 10$ (case $m=3$ ) or $\operatorname{deg}(Z) \leq 4 m-4$ (case $m \geq 4)$. We have $h^{1}\left(\mathcal{I}_{Z}(m)\right)>0$ if and only if there is a scheme $W \subseteq Z$ such that one of the following cases occurs: 
(a) $\operatorname{deg}(W)=m+2$ and $W$ is contained in a line;

(b) $\operatorname{deg}(W)=2 m+2$ and $W$ is contained in a conic;

(c) $\operatorname{deg}(W)=3 m$ and $W$ is the complete intersection of a curve of degree 3 and a curve of degree $m$;

(d) $\operatorname{deg}(W)=3 m+1$ and $W$ is contained in a degree 3 curve;

(e) $\operatorname{deg}(W)=4 m-4$ and $W$ is the complete intersection of a degree 4 curve and a degree $m-1$ curve.

Proof. Let $T$ be the degree $\leq 4$ curve listed in cases (a), (b), (c), (d), (e). In cases (a), (b), (c), (d), (e) we have $h^{1}\left(\mathcal{I}_{W}(m)\right)>0$ either because $\operatorname{deg}(W)>$ $h^{0}\left(T, \mathcal{O}_{T}(m)\right)$ (cases (a), (b), (d)) or because $W$ is a complete intersection, say $W=T \cap T_{1}$, and we may use the Koszul complex induced by the equations of the curves $T$ and $T_{1}$. Hence the "if" part follows from Lemma 2.

Now assume $h^{1}\left(\mathcal{I}_{Z}(m)\right)>0$. Fix any subscheme $W \subseteq Z$ such that $h^{1}\left(\mathcal{I}_{W}(m)\right)$ $=1$ and $h^{1}\left(\mathcal{I}_{Z^{\prime}}(m)\right)=0$ for every $Z^{\prime} \subsetneq W$ (to prove the existence of $W$ it is essential to work over an algebraically closed base field). Therefore $h^{1}\left(\mathcal{I}_{W}(t)\right)=0$ for every $t>m$. Set $d:=\operatorname{deg}(W)$. First assume $d \leq 3 m$ and $d \geq 9$. In the set-up of [8]. Corollaire 2, take $\tau:=m$ and $s:=3$. We have $\tau \geq(3-3)+d / 3$. By [8], Corollaire 2, we get that either $W$ is the complete intersection of a cubic curve and a degree $m$ curve (and hence $W=Z$ and $\operatorname{deg}(Z)=3 m$ ) or there is $t \in\{1,2\}$ and a degree $t$ curve $F \subset \mathbb{P}^{2}$ such that $\operatorname{deg}(F \cap W) \geq t m+t(3-t)$. Now assume $d \leq 8$. Adapt the case of a reduced set done, for instance, in [9], p. 715 .

Now assume $d \geq 3 m+1$ and $d \geq 16$. Since $d \geq 16$, we may apply the case $s=4, \tau=m$ of [8], Corollaire 2 (we may apply it, because $d \leq 4 m-4$, i.e., $\tau=m \geq 1+d / 4=s-3+d / s)$. We get that either $W$ is the complete intersection of a degree 4 curve and a degree $m-1$ curve (case (e)) or there is $t \in\{1,2,3\}$ and $W_{t} \subseteq W$ with $W_{t}$ contained in a degree $t$ curve and either $\operatorname{deg}\left(W_{t}\right)=t m+2$ (case $t \in\{1,2\})$ or $t=3$ and $3 m \leq \operatorname{deg}\left(W_{3}\right) \leq 3 m+1$. If $t=1$ (resp. $t=2$ ), then we are in case (a) (resp. (b)). If $t=3$ and $\operatorname{deg}\left(W_{3}\right)=3 m+1$, then we are in case (d). Hence we may assume $\operatorname{deg}\left(W_{3}\right)=3 \mathrm{~m}$. Let $E$ be a degree 3 curve containing $W_{3}$. To prove that we are in case (c) (taking $W_{3}$ as the subscheme of $Z$ ) it is sufficient to prove that $W_{3}$ is the complete intersection of $E$ and a degree $m$ plane curve. Since $W \cap E \supseteq W_{3}$ either we are in case (d) or $W \cap E=W_{3}$. Hence we may assume $W \cap E=W_{3}$ (as schemes). Since $\operatorname{deg}\left(\operatorname{Res}_{E}(W)\right)=\operatorname{deg}(W)-\operatorname{deg}\left(W_{3}\right) \leq m-4$, we have $h^{1}\left(\mathcal{I}_{\operatorname{Res}_{E}(W)}(m-1)\right)=0$. 
Hence Remark 2 gives $h^{1}\left(\mathcal{I}_{W_{3}}(m)\right)>0$. Apply the case $d \leq 3 m$ just done to $W_{3}$.

Now assume $d \leq 15$, and $3 m+1 \leq d \leq 4 m-4$. Hence $m=3$ and $10 \leq$ $d \leq 11$. Since $\operatorname{deg}(Z) \leq 10$ if $m=3$, it is sufficient to do the case $m=3$ and $d=10$. Since $\operatorname{deg}(W)=10=h^{0}\left(\mathcal{O}_{\mathbb{P}^{2}}(3)\right)$, we have $h^{0}\left(\mathcal{I}_{W}(3)\right)=h^{1}\left(\mathcal{I}_{W}(3)\right)$. Hence we are in case(d).

Lemma 3. Fix integers $r \geq 2$ and $m>1$. Let $Z \subset \mathbb{P}^{r}$ be a zerodimensional scheme such that $\operatorname{deg}(Z) \leq \max \{3 m+1,3 m+r-2\}$. If $r>$ 2 assume that $Z$ spans $\mathbb{P}^{r}$ and that the union of the non-reduced connected components of $Z$ has degree $\leq 2 m+1$. We have $h^{1}\left(\mathcal{I}_{Z}(m)\right)>0$ if and only if there is a scheme $W \subseteq Z$ as one of the schemes in the following list:

(a) $\operatorname{deg}(W)=m+2$ and $W$ is contained in a line;

(b) $\operatorname{deg}(W)=2 m+2$ and $W$ is contained in a plane conic;

(c) $\operatorname{deg}(W)=3 m$ and $W$ is the complete intersection of a degree 3 plane curve and a degree $m$ hypersurface;

(d) $\operatorname{deg}(W)=3 m+1$ and $W$ is contained in a plane cubic.

Proof. The "if " part follows from Lemma 2 and the cohomology of low degree plane curves. Now assume $h^{1}\left(\mathcal{I}_{Z}(m)\right)>0$. If $m=1$, then $\operatorname{deg}(Z) \leq$ $r+1$. Since $Z$ spans $\mathbb{P}^{r}$ and any degree $r$ subscheme of $\mathbb{P}^{r}$ is contained in a hyperplane, we have $\operatorname{deg}(Z)=r+1$. Since $h^{0}\left(\mathcal{I}_{Z}(1)\right)=0$ and $\operatorname{deg}(Z)=r+1$, we have $h^{1}\left(\mathcal{I}_{Z}(1)\right)=0$. Hence Proposition 3 is true if $m=1$. We use induction on $r$. For fixed $r$ we use induction on $m$, starting the induction with the case $m=1$ just done. In the case $r=2$ use Proposition 1 .

Now assume $r>2$. Let $H \subset \mathbb{P}^{r}$ be a hyperplane such that $\operatorname{deg}(Z \cap H)$ is maximal. Since $Z$ spans $\mathbb{P}^{r}$, we have $\operatorname{deg}(Z \cap H)<\operatorname{deg}(Z)$ and hence $\operatorname{deg}(Z \cap H) \leq 3 m+\operatorname{dim}(H)-2$. We have $h^{1}\left(H, \mathcal{I}_{Z \cap H}(m)\right)=h^{1}\left(\mathbb{P}^{r}, \mathcal{I}_{Z \cap H}(m)\right)$. Hence if $h^{1}\left(H, \mathcal{I}_{Z \cap H}(m)\right)>0$, then we may take $W \subseteq Z \cap H$ by the inductive assumption on $r$. Hence we may assume $h^{1}\left(H, \mathcal{I}_{Z \cap H}(m)\right)=0$. Remark 2 implies $h^{1}\left(\mathcal{I}_{\operatorname{Res}_{H}(Z)}(m-1)\right)>0$. By the inductive assumption on $m$ there is $W^{\prime} \subseteq \operatorname{Res}_{H}(Z)$ satisfying one of the assumptions (a), (b), (c) or (d) of Proposition 3 with respect to the integer $m^{\prime}:=m-1$. In particular there is a plane $M \subset \mathbb{P}^{r}$ such that $W^{\prime} \subset M$ and $\operatorname{deg}\left(W^{\prime}\right) \geq m+1$, with strict inequality, unless $W^{\prime}$ is contained in a line. Every zero-dimensional scheme with degree at most $r$ is contained in a hyperplane. Since $Z$ is not contained in a hyperplane, there is a hyperplane $U \subset \mathbb{P}^{r}$ such that $W^{\prime} \subset U$ and either 
$\operatorname{deg}(Z \cap U)-\operatorname{deg}\left(W^{\prime}\right) \geq r-2$ (case $W^{\prime}$ non collinear) or $\operatorname{deg}(Z \cap U) \geq m+r-1$ (case $W^{\prime}$ collinear). We took $H$ so that $\operatorname{deg}(Z \cap H) \geq \operatorname{deg}(Z \cap U)$. We have $\operatorname{deg}(Z)=\operatorname{deg}(Z \cap H)+\operatorname{deg}\left(\operatorname{Res}_{H}(Z)\right)$ (first part of Remark 2). Since $\operatorname{deg}(Z)<$ $2 m+2+2(r-2)$, we get that $W^{\prime}$ is collinear. Call $D$ the line spanned by $W^{\prime}$ and set $W^{\prime \prime}:=D \cap Z$. If $W^{\prime \prime} \neq W^{\prime}$, then $\operatorname{deg}\left(W^{\prime \prime}\right) \geq m+2$ and hence we are in case (a). Therefore we may assume $W=W^{\prime}$. If $h^{1}\left(\mathcal{I}_{Z \cap U}(m)\right)>0$, then we may take as $W$ a subscheme of $Z \cap U$. Therefore we may assume $h^{1}\left(\mathcal{I}_{Z \cap U}(m)\right)=0$. Therefore $h^{1}\left(\mathcal{I}_{\operatorname{Res}_{U}(Z)}(m-1)\right)>0$. Hence there is $A \subseteq$ $\operatorname{Res}_{U}(Z)$ as in one of the cases (a), (b), (c), (d) for the integer $m-1$. As in the case with $H$ we get that $A$ is collinear, $\operatorname{deg}(A)=m+1$ and $A=Z \cap D^{\prime}$ with $D^{\prime}$ a line. First assume $D=D^{\prime}$. Let $E$ be the union of all non-reduced connected components $A$ of $Z$ with $A_{\text {red }} \in D$, but with $A \nsubseteq D$. Let $F$ be the union of the connected components of $Z$ contained in $D$ (among them there are all the reduced connected components of $Z$ contained in $D)$. We have $\operatorname{Res}_{U}(Z)=$ $\operatorname{Res}_{U}(Z \backslash F)$ and hence $\operatorname{Res}_{U}(Z) \cap D=\operatorname{Res}_{U}(E) \cap D$. Since $\operatorname{Res}_{U}(E) \subseteq E$, we have $\operatorname{deg}\left(D \cap \operatorname{Res}_{U}(E) \cap D\right) \leq \operatorname{deg}(E \cap D)$. Therefore $m+1 \leq \operatorname{deg}(E \cap D)$. Since $2 m+1 \geq \operatorname{deg}(E)=\operatorname{deg}(E \cap D)+\operatorname{deg}\left(\operatorname{Res}_{U}(E)\right) \geq \operatorname{deg}(E \cap D)+m+1$, we get a contradiction. Therefore $D \neq D^{\prime}$. If $D \cap D^{\prime} \neq \emptyset$, then we are in case (b). Hence we may assume $D \cap D^{\prime}=\emptyset$.

First assume $r=3$. Take a smooth quadric $Q$ containing the two disjoint lines $D$ and $D^{\prime}$, say as lines of type $(1,0)$. Since $\operatorname{deg}\left(\operatorname{Res}_{Q}(Z)\right) \leq m-1$, we have $h^{1}\left(\mathcal{I}_{\operatorname{Res}_{Q}(Z)}(m-2)\right)=0$. Hence Remark 2 gives $h^{1}\left(Q, \mathcal{I}_{Z \cap Q}(m)\right)>0$. We have $h^{1}\left(Q, \mathcal{I}_{Z \cap Q}(m)\right)=h^{1}\left(\mathcal{I}_{Z \cap Q}(m)\right)$. See $D \cup D^{\prime}$ as a Cartier divisor of $Q$. Since $\operatorname{deg}(Z \cap D)=\operatorname{deg}\left(Z \cap D^{\prime}\right)=m+1$, we have $h^{i}\left(Q, \mathcal{I}_{Z \cap\left(D \cup D^{\prime}\right)}(m)\right)=0$, $i=0,1$. Hence $h^{1}\left(Q, \mathcal{I}_{Z \cap Q}(m)\right)=h^{1}\left(Q, \mathcal{I}_{\operatorname{Res}_{D \cup D^{\prime}}(Z \cap Q)}(m-2, m)\right)$. Therefore $h^{1}\left(Q, \mathcal{I}_{\operatorname{Res}_{D \cup D^{\prime}}(Z \cap Q)}(m-2, m)\right)>0$ and so $h^{1}\left(Q, \mathcal{I}_{\operatorname{Res}_{D \cup D^{\prime}}(Z \cap Q)}(m-2, m-\right.$ $2))>0$. Hence $\operatorname{deg}\left(\operatorname{Res}_{D \cup D^{\prime}}(Z \cap Q)\right) \geq m$. Hence $\operatorname{deg}(Z) \geq 3 m+2$, contradiction.

Now assume $r>3$. Let $H^{\prime}$ be any hyperplane containing $D \cup D^{\prime}$ and (among these hyperplanes) with maximal $\operatorname{deg}\left(Z \cap H^{\prime}\right)$. Since $Z$ spans $\mathbb{P}^{r}$, we have $\operatorname{deg}\left(Z \cap H^{\prime}\right) \geq 2 m+r-3$. Hence $\operatorname{deg}\left(\operatorname{Res}_{H^{\prime}}(Z)\right) \leq m$. Hence $h^{1}\left(\mathcal{I}_{\left.\operatorname{Res}_{H^{\prime}}(Z)\right)}(m-1)\right)=0$. Hence Remark 2 gives $h^{1}\left(\mathcal{I}_{Z \cap H^{\prime}}(m)\right) \geq e$. Apply the inductive assumption on $r$ to the scheme $Z \cap H^{\prime}$.

Proof of Theorem 1. First assume $\operatorname{deg}(Z) \leq 3 m+\rho-2$. With no loss of generality we may assume $r=\rho$. Since the case $r=2$ is true by Proposition 1 , we may assume $r>2$ and use induction on $r$. Since the case $e=1$ is true by Proposition 1 we may assume $e \geq 2$ and use induction on $e$. Since $h^{1}\left(\mathcal{I}_{Z}(m)\right) \geq e \geq e-1$, there is $W \subseteq Z$ with $W$ one of the schemes in the 
following list:

(i) $\operatorname{deg}(W)=m+e$ and $W$ is contained in a line $L$;

(ii) $\operatorname{deg}(W)=2 m+e$ and $W$ is contained in a plane conic;

(iii) $e \geq 3, \operatorname{deg}(W)=2 m+e+1$, and there are an integer $f \in\{1, \ldots, e-2\}$ and lines $L_{1}, L_{2}$, such that $L_{1} \cap L_{2}=\emptyset, W \subset L_{1} \cup L_{2}, \operatorname{deg}\left(L_{1} \cap W\right)=m+1+f$ and $\operatorname{deg}\left(L_{2} \cap W\right)=m+e-f$.

(a) First assume that $W$ is as in cases (i) or (ii) or that $r \geq 4$ and that we are as in case (iii). Let $H \subset \mathbb{P}^{r}$ be any hyperplane containing $\langle W\rangle$, where \langle\rangle denote the linear span, and with maximal $u:=\operatorname{deg}(Z \cap H)$. Since $Z$ spans $\mathbb{P}^{r}$, we have $\operatorname{deg}(Z \cap H)<\operatorname{deg}(Z) \leq 3 m+r-2$. Since $Z$ spans $\mathbb{P}^{r}$, the maximality property of the integer $u$ gives $\langle Z \cap H\rangle=H$. If $A \subseteq Z \cap H$ is a connected component of $Z \cap H$, which is not reduced, then the connected component $B$ of $Z$ with $B_{\text {red }}=A_{\text {red }}$ contains $A$ and hence $\operatorname{deg}(A) \leq \operatorname{deg}(B)$. Therefore we may use induction on $r$ (for an arbirary integer $e$ ) for the scheme $Z \cap H \subset H$. Since $Z \cap H \supseteq W$, we have $h^{1}\left(H, \mathcal{I}_{Z \cap H}(m)\right) \geq e-1$. If $h^{1}\left(H, \mathcal{I}_{Z \cap H}(m)\right) \geq$ $e$, then we may use the inductive assumption on $r$. Hence we may assume that $h^{1}\left(H, \mathcal{I}_{Z \cap H}(m)\right)=e-1$. By the residual exact sequence (1) we get $h^{1}\left(\mathcal{I}_{\operatorname{Res}_{H}(Z)}(m-1)\right)>0$. Therefore $\operatorname{deg}\left(\operatorname{Res}_{H}(Z)\right) \geq m+1([6$, Lemma 34]). Since $Z \cap H$ spans $H$, we have $u \geq r-1-\operatorname{dim}(\langle W\rangle)+\operatorname{deg}(W)$. Hence $\operatorname{deg}(Z) \geq$ $m+r-\operatorname{dim}(\langle W\rangle)+\operatorname{deg}(W)$. In case (ii) we get $\operatorname{deg}(Z) \geq m+r-2+2 m+e=$ $3 m+r-2+e$, a contradiction. In case (iii) we get $\operatorname{deg}(Z) \geq m+r-3+2 m+e+1$, a contradiction. Now assume that we are in case (i). We may assume $W=Z \cap L$ (as schemes), because otherwise $\operatorname{deg}(Z \cap L) \geq m+1+e$ and we may take as new $W$ a subscheme of $Z \cap L$ with degree $m+1+e$. Since $\operatorname{deg}(Z \cap H) \geq m+e+r-2$, we have $\operatorname{deg}\left(\operatorname{Res}_{H}(Z)\right) \leq 2 m-e-1$. By [6, Lemma 34] there is a line $D \subset \mathbb{P}^{r}$ $\operatorname{such}$ that $\operatorname{deg}\left(D \cap \operatorname{Res}_{H}(Z)\right) \geq m+1$.

(a1) First assume $D \cap L \neq \emptyset$ and $D \neq L$. Therefore $\langle D \cup L\rangle$ is a plane. We are in case $(\mathrm{b})$.

(a2) Now assume $D \cap L=\emptyset$. If $D \cap Z \supsetneq D \cap \operatorname{Res}_{H}(L)$ (or if $\operatorname{deg}(Z \cap D) \geq$ $m+2$ ), then we are as in case (c) with $f=e-1, L_{1}=L$ and $L_{2}=D$. Now assume $\operatorname{deg}(D \cap Z)=m+1$. Let $M:=\langle D \cup L\rangle$ be the 3-dimensional linear space. First assume $r=3$. Let $Q \subset M$ be any smooth quadric containing $D \cup L$. Since $\operatorname{deg}(Q \cap Z) \geq 2 m+1+e$, we have $\operatorname{deg}\left(\operatorname{Res}_{Q}(Z)\right) \leq m-1-e$. Hence $h^{1}\left(\mathcal{I}_{\operatorname{Res}_{Q}(Z)}(m-2)\right)=0$. Remark 2 gives $h^{1}\left(Q, \mathcal{I}_{Z \cap Q}(m)\right) \geq e$. Call $(1,0)$ the type of the ruling of $Q$ containing $D$ and $L$. Since $\operatorname{deg}\left(\operatorname{Res}_{D \cup L}(Q \cap Z)\right) \leq$ $m-1-e$, we have $h^{1}\left(Q, \mathcal{I}_{\operatorname{Res}_{D \cup L}(Q \cap Z)}(m-2, m)\right) \leq h^{1}\left(Q, \mathcal{I}_{\operatorname{Res}_{D \cup L}(Q \cap Z)}(m-\right.$ 
$2, m-2))=0$. Therefore Remark 2 gives $h^{1}\left(Q, \mathcal{I}_{Z \cap Q}(m)\right) \leq h^{1}\left(D, \mathcal{I}_{Z \cap D}(m)\right)+$ $h^{1}\left(L, \mathcal{I}_{L \cap Z}(m)\right)=0+e-1$, contradicting the inequality $h^{1}\left(Q, \mathcal{I}_{Z \cap Q}(m)\right) \geq e$ just proved. Now assume $r \geq 4$. Let $N \subset \mathbb{P}^{r}$ be any hyperplane containing $M$ and with maximal $\operatorname{deg}(Z \cap N)$ among all hyperplanes containing $M$. Since $Z$ is non-degenerate, we have $\operatorname{deg}(Z \cap N) \geq \operatorname{deg}(Z \cap M)+r-4 \geq 2 m+e+r-3$. By induction on $r$ we get $h^{1}\left(N, \mathcal{I}_{N \cap Z}(m)\right)=e-1$. Since $\operatorname{deg}\left(\operatorname{Res}_{N}(Z)\right) \leq$ $\operatorname{deg}(Z)-\operatorname{deg}(Z \cap(L \cup D)) \leq 3 m+r-2-2 m-e-r+3=m+1-e$, we have $h^{1}\left(\mathcal{I}_{\operatorname{Res}_{N}(Z)}(m)\right)=0$. Remark 2 gives $h^{1}\left(\mathcal{I}_{Z}(m)\right) \leq e-1$, a contradiction.

(a3) Now assume $D=L$. Let $E$ be the union of all non-reduced connected components $A$ of $Z$ with $A_{\text {red }} \in L$, but with $A \nsubseteq L$. Let $F$ be the union of the connected components of $Z$ contained in $L$ (among them there are all the reduced connected components of $Z$ contained in $F$. We have $\operatorname{Res}_{H}(Z)=$ $\operatorname{Res}_{H}(Z \backslash F)$ and hence $\operatorname{Res}_{H}(Z) \cap L=\operatorname{Res}_{H}(E) \cap L$. Since $\operatorname{Res}_{H}(E) \subseteq E$, we have $\operatorname{deg}\left(L \cap \operatorname{Res}_{H}(E) \cap L\right) \leq \operatorname{deg}(E \cap L)$. Therefore $m+1 \leq \operatorname{deg}(E \cap L)$. Since $2 m+1 \geq \operatorname{deg}(E)=\operatorname{deg}(E \cap H)+\operatorname{deg}\left(\operatorname{Res}_{H}(E)\right) \geq \operatorname{deg}(E \cap L)+m+1$, we get a contradiction.

(b) Now assume $r=3, e \geq 3$, and that $W$ is as in case (iii). If either $W \cap$ $L_{1} \neq Z \cap L_{1}$ or $W \cap L_{2} \neq Z \cap L_{2}$, then we are in case (c) with as integer $f$ either $f$ or $f+1$. Therefore we may assume $Z \cap\left(L_{1} \cup L_{2}\right)=W$. Let $Q$ be any smooth quadric containing $L_{1} \cup L_{2}$. We have $\operatorname{deg}\left(\operatorname{Res}_{Q}(Z)\right)=\operatorname{deg}(Z)-\operatorname{deg}(Z \cap Q) \leq$ $\operatorname{deg}(Z)-\operatorname{deg}\left(Z \cap L_{1} \cup L_{2}\right) \leq m-1-e$. Therefore $h^{1}\left(\mathcal{I}_{\operatorname{Res}_{Z}(Q)}(m-2)\right)=0([6$, Lemma 34]). Remark 2 gives $h^{1}\left(Q, \mathcal{I}_{Q \cap Z}(m)\right)=0$. Set $T:=L_{1} \cup L_{2}$ seeing as a divisors of $Q$, say of type $(2,0)$. Since $T \cap Z=W$, we have $h^{1}\left(T, \mathcal{I}_{T \cap Z}(m)\right)=$ $e-1$. We have $\operatorname{deg}\left(\operatorname{Res}_{T}(Z \cap Q)\right)=\operatorname{deg}(Z \cap Q)-\operatorname{deg}(Z \cap T) \leq m-1-e$ and hence $h^{1}\left(Q, \mathcal{I}_{\operatorname{Res}_{T}(Z \cap Q)}(m-2, m)\right) \leq h^{1}\left(Q, \mathcal{I}_{\operatorname{Res}_{T}(Z \cap Q)}(m-2, m-2)\right)=0$. The residual exact sequence of the inclusion $T \subset Q$ gives a contradiction.

(c) Now assume $\operatorname{deg}(Z) \geq 3 m+r-1$, but $\operatorname{deg}(Z) \leq 3 m+e-2$. Taking the linear span $\langle Z\rangle$ of $Z$ instead of $\mathbb{P}^{r}$, we may assume $\langle Z\rangle=\mathbb{P}^{r}$. Since the case $r=2$ is true by Proposition 1 we may assume $r>2$ and use induction on $r$. Since the case $e=1$ is true by Lemma 3 we may assume $e \geq 2$ and use induction on $e$. Take $H, W$ as in the first part of the proof. In case (ii) (resp. case (iii) with $r>3$ ) of step (a) we use that $\operatorname{deg}\left(\operatorname{Res}_{H}(W)\right) \leq m-2$ (resp. $m-3)$. Now assume that we are in case (i). We have $\operatorname{deg}(Z)-\operatorname{deg}(Z \cap H) \leq$ $3 m+e-2-(m+1+e)-(r-2) \leq 2(m-1)+1$. As in the proof of Theorem 1 quoting [6, Lemma 34] we get a line $D$ with $\operatorname{deg}\left(D \cap \operatorname{Res}_{H}(Z)\right) \geq m+1$. The assumption on the non-reduced connected components of $Z$ gives $D \neq L$. If $D \cap L \neq \emptyset$, then we get $\operatorname{deg}(Z \cap(D \cup L)) \geq 2 m+1+e$ and hence we are in case (b). If $D \cap L=\emptyset$, we are in case ( c) with $f=e-1, L_{1}=L$ and $L_{2}=D$. If $r=3$ and we are in case (iii), then step (b) gives a contradiction, unless 
$\left(L_{1} \cup L_{2}\right) \cap Z \supsetneq W$, which gives case (c) of Theorem 1 .

\section{Acknowledgements}

The author was partially supported by MIUR and GNSAGA of INdAM (Italy).

\section{References}

[1] E. Ballico, Finite subsets of projective spaces with bad postulation in a fixed degree, Beitr. Algebra Geom. 54 (2013), 81-103 . DOI $10.1007 / \mathrm{s} 13366-012-0104-8$

[2] E. Ballico, Generalized Hamming weights of codes coming from smooth plane curves, Afrika Matematika 24 (2013), no. 4, pp 565-569

[3] E. Ballico, Defective curvilinear subschemes in projective spaces, J. Pure Appl. Math. 81 (2012), no. 1, 49-53.

[4] E. Ballico, Zero-dimensional schemes in the plane, Sarajevo J. Math. (to appear).

[5] E. Ballico, A. Ravagnani, The dual geometry of Hermitian two-point codes. Discrete Mathematics, 313 (2013), 23, 2687 - 2695.

[6] A. Bernardi, A. Gimigliano and M. Idà, Computing symmetric rank for symmetric tensors, J. Symbolic Comput. 46 (2011), no. 1, 34-53.

[7] A. Couvreur, The dual minimum distance of arbitrary dimensional algebraic-geometric codes. J. of Algebra 350 (2012), no. 1, 84-107 (2012).

[8] Ph. Ellia, and Ch. Peskine, Groupes de points de $\mathbf{P}^{2}$ : caractère et position uniforme.Algebraic geometry (L'Aquila, 1988), 111-116, Lecture Notes in Math., 1417, Springer, Berlin, 1990.

[9] Griffiths Ph., Harris, J.: Principles of Algebraic Geometry. John Wiley \& Sons, New York, 1978.

[10] G. M. Hana and T. Johnsen, Scroll codes,. Des. Codes Cryptogr. 45 (2007), no. $3,365-377$ (2007). 
[11] S. H. Hansen, Error-correcting codes from higher-dimensional varieties, Finite Fields Appl. 7 (2001), no. 4, 530-552.

[12] T. Johnsen and N. H. Rasmussen, Scroll codes over curves of higher genus, Appl. Algebra Engrg. Comm. Comput. 21 (2010), 397-415 (2010). 
